\title{
Outage analysis of ZFB-MRT/MRC Underlay Two-Way Relay Systems
}

\author{
Yun CAO and Chintha Tellambura, Fellow, IEEE
}

\begin{abstract}
We analyse the end-to-end (E2E) outage probabilities (OPs) of zero-forcing beamforming and maximal-ratiotransmission/combining (ZFB-MRT/MRC) for an underlay network, which consists of a single-antenna fixed-gain amplify-andforward (AF) two-way relay and two multi-antenna terminals. Assuming both path loss effects and small-scale fading and considering both secondary-to-primary (S2P) and primary-tosecondary (P2S) interferences, the exact and asymptotic E2E OPs are derived.
\end{abstract}

Index Terms - amplify-and-forward relaying, cognitive radio, outage probability, underlay, ZFB-MRT/MRC

\section{INTRODUCTION}

The cognitive underlay concept yields improved spectral efficiency and spectrum utilization. However, these improvements may be limited due to secondary transmitters having to reduce their transmit power to comply with the S2P interference constraint and secondary receivers are being subject to P2S interferences. To mitigate the first effect, beamforming and one-way relays were used and their OPs were analysed [1]-[3]. However, the performance of beamforming for underlay two-way AF relay networks considering both S2P and P2S interferences has not been analysed.

Therefore, we study an underlay two-way AF relay network, which consisting of two terminals $\left(\mathrm{SU}_{1}\right.$ and $\left.\mathrm{SU}_{2}\right)$ with $M_{1}$ and $M_{2}$ antennas, respectively, and a single-antenna half-duplex relay $(\mathrm{R})$ co-exists with a primary transmitter $(\mathrm{A})$ and receiver (B) (Fig. 1). A similar system configuration but with one-way relay was studied in [3]. We consider AF relaying due to its advantages of low complexity and short processing time [4]. In our study, however, the relay is limited to a single-antenna device for two reasons: (1) multi-antenna relays may face the size and cost constraints [5], and (2) the analysis of the multiantenna relay case is beyond the scope of this letter.

In this configuration, the $\mathrm{SU}_{j} \rightarrow \mathrm{B}$ and $\mathrm{A} \rightarrow \mathrm{SU}_{j}$ interference signals are mitigated via ZFB-MRT and ZFB-MRC, respectively. However, since mutual information exchange between $\mathrm{SU}_{1}$ and $\mathrm{SU}_{2}$ requires two time slots, the $\mathrm{A} \rightarrow \mathrm{R}$ interference in time slot one will propagate to $\mathrm{SU}_{1}$ and $\mathrm{SU}_{2}$ in time slot two. To the best of our knowledge, a comprehensive analysis considering both $\mathrm{P} 2 \mathrm{~S}$ and $\mathrm{S} 2 \mathrm{P}$ interference links (Fig. 1) has not yet been furnished. Therefore, considering all those effects, we derive both the exact and asymptotic E2E OPs and important insights are: (1) The location of the relay

Yun CAO and Chintha Tellambura are with the Department of Electrical and Computer Engineering, University of Alberta, Edmonton, AB, Canada T6G 2V4 (Email: \{cao7, chintha\}@ece.ualberta.ca). significantly impacts the outage; (2) As the secondary transmit power $P_{s} \rightarrow \infty$, the diversity order is zero if the interference threshold $I_{t h}$ is finite; (3) As $P_{s} \rightarrow \infty$, if $\frac{I_{t h}}{P_{s}}$ is constant, a diversity of order $\left(\min \left(M_{1}, M_{2}\right)-1\right)$ is achieved.

Notations: $(\bullet)^{*},(\bullet)^{T},\|\bullet\|_{F}$, and $\mathbb{E}\{\bullet\}$ represent complex conjugation, transpose, Frobenius norm, and expectation, respectively. $\boldsymbol{I}$ is the identity matrix. Regularized incomplete Gamma function $P(a, b)=\frac{\int_{0}^{b} t^{a-1} e^{-t} d t}{\int_{0}^{\infty} t^{a-1} e^{-t} d t}$, incomplete Beta function $B(x ; \alpha, \beta)$ [6, Eq. (8.391)] and Beta function $B(\alpha, \beta)=B(1, \alpha, \beta)$. The probability density function (PDF) and cumulative distribution function (CDF) of $X$ are $f_{X}(x)$ and $F_{X}(x)$. If $f_{X}(x)=\lambda e^{-\lambda x}, x \geq 0$, we write $X \sim \operatorname{Exp}(\lambda) . X \sim \operatorname{Gamma}(k, \theta)(k>0, \theta>0)$, if $F_{X}(x)=P\left(k, \frac{x}{\theta}\right), x>0 . X \sim \operatorname{Beta}(\alpha, \beta)(\alpha>0, \beta>0)$, if $f_{X}(x)=\frac{x^{\alpha-1}(1-x)^{\beta-1}}{B(\alpha, \beta)}, 0 \leq x \leq 1 . \mathcal{C N}\left(\mu, \sigma^{2}\right)$ denotes complex Gaussian with mean $\mu$ and variance $\sigma^{2} . \mathcal{K}_{\nu}(x)$ is the modified Bessel function of the second type of order $\nu . \mathbb{C}$ is the set of complex numbers.

\section{Underlay RELAy Systems And Signal Flow}

\section{A. System Configuration and Signal Flow}

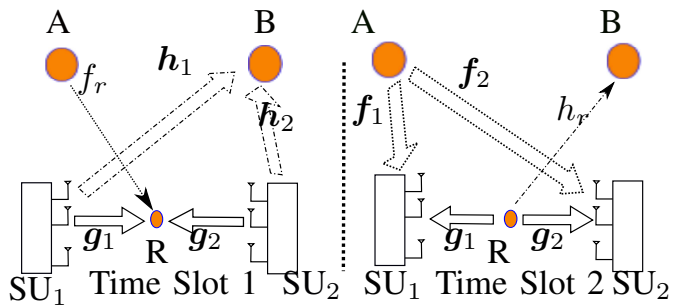

Fig. 1. Underlay Two-way Relay Model.

As mentioned before, in our underlay cognitive setup (Fig. 1), the two secondary multi-antenna transceivers $\mathrm{SU}_{j}$ have $M_{j} \geq 2$ antennas $(j=1,2)$, and the half-duplex singleantenna AF relay is $\mathrm{R}$. No direct link is assumed between $\mathrm{SU}_{1}$ and $\mathrm{SU}_{2}$. As in [2], [3], perfect time synchronization is assumed between the primary and secondary networks. Thus, our results characterize the worst-case interference scenario.

This work considers both path loss and small-scale fading. Consider two nodes $\mathrm{x}$ and $\mathrm{y}$ with $n_{x}$ and $n_{y}$ antennas at a distance $d_{\mathrm{x}, \mathrm{y}}$ in Fig. 1. The channel from $\mathrm{x}$ to $\mathrm{y}$ is thus denoted by a $\mathbb{C}^{n_{y} \times n_{x}}$ matrix with independent and identical distributed $\mathcal{C N}\left(0, \lambda_{\mathrm{x}, \mathrm{y}}\right)$ entries, where $\lambda_{\mathrm{x}, \mathrm{y}}$ accounts for the path loss and satisfies $\lambda_{\mathrm{x}, \mathrm{y}} \propto d_{\mathrm{x}, \mathrm{y}}^{-\omega}$, where $\omega$ is the path loss exponent. Therefore, vectors $\boldsymbol{g}_{j} \in \mathbb{C}^{M_{j} \times 1}, \boldsymbol{h}_{j} \in \mathbb{C}^{M_{j} \times 1}$ and $\boldsymbol{f}_{j} \in \mathbb{C}^{M_{j} \times 1}(j=1,2)$ are the reciprocal $\mathrm{SU}_{j} \leftrightarrow \mathrm{R}$ channel, the $\mathrm{SU}_{j} \rightarrow \mathrm{B}$ and $\mathrm{A} \rightarrow \mathrm{SU}_{j}$ interference channels with $g_{j_{i}} \sim$ 
$\mathcal{C N}\left(\mathbf{0}, \lambda_{j}\right), h_{j_{i}} \sim \mathcal{C N}\left(\mathbf{0}, \lambda_{\mathrm{SU}_{j}, \mathrm{~B}}\right)$ and $f_{j_{i}} \sim \mathcal{C N}\left(\mathbf{0}, \lambda_{\mathrm{A}, \mathrm{SU}_{j}}\right)$ $\left(i=1,2, \ldots, M_{j}\right)$, respectively. $h_{r} \sim \mathcal{C N}\left(0, \lambda_{3}\right)$ and $f_{r} \sim$ $\mathcal{C N}\left(0, \lambda_{0}\right)$ are the $\mathrm{R} \rightarrow \mathrm{B}$ and $\mathrm{A} \rightarrow \mathrm{R}$ interference channels, respectively. Here, $\lambda_{0}=\lambda_{\mathrm{A}, \mathrm{R}}, \lambda_{j}=\lambda_{\mathrm{SU}_{j}, \mathrm{R}}(j=1,2)$, and $\lambda_{3}=\lambda_{\mathrm{R}, \mathrm{B}} \cdot \boldsymbol{m}_{j}, \boldsymbol{d}_{j} \in \mathbb{C}^{M_{j} \times 1}$ are the normalized transmit and receive beamforming vectors at $\mathrm{SU}_{j}(j=1,2)$, respectively.

In [1]-[3], channel gains of the whole network, e.g. all the secondary-to-secondary (S2S), P2S and S2P channels, are assumed known at secondary nodes. However because such full CSI requirement necessitates a large overhead, we assume that every secondary node $\mathrm{x} \in\left\{\mathrm{SU}_{1}, \mathrm{SU}_{2}\right.$, and $\left.\mathrm{R}\right\}$ obtains channel gains only of channels involving itself, e.g. $\mathrm{x} \rightarrow \mathrm{B}, \mathrm{A} \rightarrow \mathrm{x}, \mathrm{x} \rightarrow \mathrm{R} / \mathrm{SU}_{j}$ (if $\mathrm{x}=\mathrm{SU}_{j} / \mathrm{R}, j=1,2$ ), via a suitable channel estimation process [7]. With this assumption, $\mathrm{SU}_{j}$ can calculate its own beamforming vectors $\boldsymbol{m}_{j}$ and $\boldsymbol{d}_{j}(j=1,2)$, and the relay (R) could adapt its relay gain $G \in \mathbb{C}$ if $\mathrm{SU}_{j}$ communicates $\boldsymbol{m}_{j}$ to R. However, to reduce this overhead, we assume $\mathrm{SU}_{j}$ sends only the average $\left(\mathbb{E}\left\{\left|\boldsymbol{g}_{j}^{T} \boldsymbol{m}_{j}\right|^{2}\right\}\right)$ to $\mathrm{R}$, then R calculates $G$ and feeds it back to $\mathrm{SU}_{j}(j=1,2)$. Such averages are more static than the actual instant channel gains themselves, and thus we obtain a fixed-gain relay. However, our numerical comparisons with a relay using a channelassisted gain are also provided.

Without loss of generality, zero-mean complex additive white Gaussian noise (AWGN) with $N_{0}$ variance and unit symbol power are assumed. $I_{t h}$ denotes the interference temperature limit. $P_{s}$ and $P_{p}$ denote the transmit power at $\mathrm{SU}_{j}$ $(j=1,2)$ and A, respectively.

The two-way relay requires two time slots. In time slot one, $\mathrm{SU}_{j}(j=1,2)$ and $\mathrm{A}$ transmit symbols $s_{j}$ and $x^{(1)}$, respectively. Given $n_{r}$ as the noise at R, the signal $y_{r}$ and interference signal $x_{i n t}^{(1)}$ received at $\mathrm{R}$ and $\mathrm{B}$ are then given as,

$$
\begin{aligned}
y_{r} & =\sqrt{P_{s}} \boldsymbol{g}_{1}^{T} \boldsymbol{m}_{1} s_{1}+\sqrt{P_{s}} \boldsymbol{g}_{2}^{T} \boldsymbol{m}_{2} s_{2}+\sqrt{P_{p}} f_{r} x^{(1)}+n_{r}, \\
x_{i n t}^{(1)} & =\sqrt{P_{s}} \boldsymbol{h}_{1}^{T} \boldsymbol{m}_{1} s_{1}+\sqrt{P_{s}} \boldsymbol{h}_{2}^{T} \boldsymbol{m}_{2} s_{2} .
\end{aligned}
$$

In the second time slot, $\mathrm{R}$ transmits $G y_{r}$. The interference signal $x_{i n t}^{(2)}$ received at primary receiver B is given as

$$
x_{i n t}^{(2)}=h_{r} G y_{r} .
$$

Because the underlay mode requires the interference power at $\mathrm{B}$ below the interference temperature limit $\left(I_{t h}\right)$, we choose $G$ such that $\mathbb{E}\left\{\left|x_{i n t}^{(2)}\right|^{2}\right\} \leq I_{t h}$. Therefore, the fixed relay gain $G$ must satisfy,

$$
G^{2}=\frac{I_{t h}}{\mathbb{E}\left\{\left|h_{r}\right|^{2}\right\}\left[P_{s} \mathbb{E}\left\{\left|\boldsymbol{g}_{1}^{T} \boldsymbol{m}_{1}\right|^{2}\right\}+P_{s} \mathbb{E}\left\{\left|\boldsymbol{g}_{2}^{T} \boldsymbol{m}_{2}\right|^{2}\right\}+P_{p} \mathbb{E}\left\{\left|f_{r}\right|^{2}\right\}+N_{0}\right]} .
$$

Also in time slot two, A transmits $x^{(2)}$. Knowing $G$ and $\boldsymbol{g}_{j}(j=1,2), \mathrm{SU}_{j}$ can eliminate the self-interference part $\boldsymbol{g}_{j} G \boldsymbol{g}_{j}^{T} \boldsymbol{m}_{j} s_{j}$ in its received signal perfectly. After that and receive beamforming, the resulting signal $\hat{y}_{j}$ is represented as

$$
\begin{aligned}
\hat{y}_{j}= & \underbrace{\sqrt{P_{s}} G \boldsymbol{d}_{j}^{T} \boldsymbol{g}_{j} \boldsymbol{g}_{\bar{j}}^{T} \boldsymbol{m}_{\bar{j}} s_{\bar{j}}}_{\text {Signal }}+\underbrace{G \boldsymbol{d}_{j}^{T} \boldsymbol{g}_{j} n_{r}+\boldsymbol{d}_{j}^{T} \boldsymbol{n}_{j}}_{\text {Noise }} \\
& +\underbrace{\sqrt{P_{p}} G \boldsymbol{d}_{j}^{T} \boldsymbol{g}_{j} f_{r} x^{(1)}+\sqrt{P_{p}} \boldsymbol{d}_{j}^{T} \boldsymbol{f}_{j} x^{(2)}}_{\text {P2S Interference }}
\end{aligned}
$$

where $\boldsymbol{n}_{j} \in \mathbb{C}^{M_{j} \times 1}$ is the AWGN at $\mathrm{SU}_{j}$. And $\bar{j}=1$, if $j=2$, and vise versa.

\section{B. ZFB-MRT/MRC Beamforming at Transmision/Reception}

With ZFB-MRT, $\boldsymbol{m}_{j}(j=1,2)$ is determined to nullify the $\mathrm{SU}_{j} \rightarrow \mathrm{B}$ interference while maximizing the $\mathrm{SU}_{j} \rightarrow \mathrm{R}$ signal power. Consequently, $\boldsymbol{m}_{j}$ is computed as the projection of the $\mathrm{SU}_{j} \rightarrow \mathrm{R}$ channel $\boldsymbol{g}_{j}$ onto the sub-space $\Phi_{j}=\boldsymbol{I}-\frac{\boldsymbol{h}_{j}^{*} \boldsymbol{h}_{j}^{T}}{\left\|\boldsymbol{h}_{j}\right\|_{F}^{2}}$, which is orthogonal to the $\mathrm{SU}_{j} \rightarrow \mathrm{B}$ interference channel $\boldsymbol{h}_{j}$. Thus, $\boldsymbol{m}_{j}=\frac{\Phi_{j} \boldsymbol{g}_{j}^{*}}{\sqrt{\boldsymbol{g}_{j}^{T} \Phi_{j} \boldsymbol{g}_{j}^{*}}}$, where the denominator is the normalizing factor. Similarly, ZFB-MRC is employed to compute the receive beamforming vector $\boldsymbol{d}_{j}(j=1,2)$ as $\boldsymbol{d}_{j}=\frac{\Psi_{j} \boldsymbol{g}_{j}^{*}}{\sqrt{\boldsymbol{g}_{j}^{T} \Psi_{j} \boldsymbol{g}_{j}^{*}}}$, where $\Psi_{j}=\boldsymbol{I}-\frac{\boldsymbol{f}_{j}^{*} \boldsymbol{f}_{j}^{T}}{\left\|\boldsymbol{f}_{j}\right\|_{F}^{2}}$.

After receive beamforming, the processed signal has the residual accumulated interference of $x^{(1)}$, which must be considered in performance analysis.

\section{End-To-End OUtage PRobability}

In this section, the exact and asymptotic E2E OPs and the fixed relay gain $G$ are derived.

\section{A. E2E Outage Probability}

After substituting the beamforming vectors $\boldsymbol{m}_{j}$ and $\boldsymbol{d}_{j}$ into (5), the $\operatorname{SINR} S_{j}$ at $\mathrm{SU}_{j}(j=1,2)$ may be represented as

$$
S_{j}=\frac{\gamma_{\bar{j} R} \gamma_{R j}}{\gamma_{R j}\left(\gamma_{3}+1\right)+C},
$$

where $\gamma_{j R}=\bar{\gamma}\left\|\boldsymbol{g}_{j}\right\|_{F}^{2} \rho_{\boldsymbol{h}_{j}}, \rho_{\boldsymbol{h}_{j}}=1-\frac{\left|\boldsymbol{h}_{j}^{T} \boldsymbol{g}_{j}^{*}\right|^{2}}{\left\|\boldsymbol{h}_{j}\right\|_{F}^{2}\left\|\boldsymbol{g}_{j}\right\|_{F}^{2}}, \gamma_{R j}=$ $\bar{\gamma}\left\|\boldsymbol{g}_{j}\right\|_{F}^{2} \rho_{\boldsymbol{f}_{j}}, \rho_{\boldsymbol{f}_{j}}=1-\frac{\left|\boldsymbol{f}_{j}^{T} \boldsymbol{g}_{j}^{*}\right|^{2}}{\left\|\boldsymbol{f}_{j}\right\|_{F}^{2}\left\|\boldsymbol{g}_{j}\right\|_{F}^{2}}, \gamma_{3}=\gamma_{0}\left|f_{r}\right|^{2}, C=\frac{\bar{\gamma}}{G^{2}}$, $\bar{\gamma}=\frac{P_{s}}{N_{0}}$, and $\gamma_{0}=\frac{P_{p}}{N_{0}}$.

Theorem 1. The random variables $\gamma_{j R}$ and $\gamma_{R j}(j=1,2)$ are $\operatorname{Gamma}\left(M_{j}-1, \bar{\gamma} \lambda_{j}\right)$ distributed.

Proof. Define $\hat{\boldsymbol{h}}_{j}=\frac{\boldsymbol{h}_{j}}{\sqrt{\lambda_{S U_{j}, B}}}$ and $\hat{\boldsymbol{g}}_{j}=\frac{\boldsymbol{g}_{j}}{\sqrt{\lambda_{j}}}(j=1,2)$. Both $\hat{\boldsymbol{h}_{j}}$ and $\hat{\boldsymbol{g}_{j}}$ are thus $\mathcal{C N}(0,1)$ distributed and $\rho_{\boldsymbol{h}_{j}}$ remains unchanged when $\boldsymbol{h}_{j}$ and $\boldsymbol{g}_{j}$ are replaced by $\hat{\boldsymbol{h}}_{j}$ and $\hat{\boldsymbol{g}}_{j}$, respectively. It has been proven in [8] that the random variable $X=\frac{\left|\hat{\boldsymbol{h}}_{j}^{T} \hat{\boldsymbol{g}}_{j}^{*}\right|^{2}}{\left\|\hat{\boldsymbol{h}}_{j}\right\|_{F}^{2}\left\|\hat{\boldsymbol{g}}_{j}\right\|_{F}^{2}}$ is $\operatorname{Beta}\left(1, M_{j}-1\right)$ distributed and is independent from both $\hat{\boldsymbol{g}}_{j}$ and $\hat{\boldsymbol{h}}_{j}$ because $X$ is the normalized correlation between $\hat{\boldsymbol{g}}_{j}$ and a uniformly distributed variable $\boldsymbol{v}=\frac{\hat{\boldsymbol{h}}_{j}}{\left\|\hat{\boldsymbol{h}}_{j}\right\|_{F}}$. Since the Beta distribution has the property that if $X \sim \operatorname{Beta}(\alpha, \beta)$, then $1-X \sim \operatorname{Beta}(\beta, \alpha), \rho_{\boldsymbol{h}_{j}}=1-X$ is $\operatorname{Beta}\left(M_{j}-1,1\right)$-distributed.

The random variable $Y_{j}=\left\|\boldsymbol{g}_{j}\right\|_{F}^{2}$ is a sum of $M_{j}$ absolute square $\mathcal{C N}\left(0, \lambda_{j}\right)$ terms and as such is $\operatorname{Gamma}\left(M_{j}, \lambda_{j}\right)$ distributed. Then by expanding $P(s, x)=1-\sum_{i=1}^{s} \frac{x^{s-i} e^{-x}}{(s-i) !}$, given $s$ is a positive integer, the $\mathrm{CDF}$ of $\gamma_{j R}$ is derived as,

$$
\begin{aligned}
F_{\gamma_{j R}}(\gamma) & =\int_{0}^{\frac{\gamma}{\gamma}} f_{Y_{j}}(y) \mathrm{d} y+\int_{\frac{\gamma}{\gamma}}^{\infty}\left(\frac{\gamma}{\bar{\gamma} y}\right)^{M_{j}-1} \frac{y^{M_{j}-1} e^{-\frac{y}{\lambda_{j}}}}{\int_{0}^{\infty} t^{M_{j}-1} e^{-t} d t \lambda_{j}^{M_{j}}} \mathrm{~d} y \\
& =1-\sum_{i=1}^{M_{j}} \frac{\gamma^{M_{j}-i} e^{-\frac{\gamma}{\bar{\gamma} \lambda_{j}}}}{\left(\bar{\gamma} \lambda_{j}\right)^{M_{j}-i}\left(M_{j}-i\right) !}+\frac{\gamma^{M_{j}-1} e^{-\frac{\gamma}{\bar{\gamma} \lambda_{j}}}}{\left(\bar{\gamma} \lambda_{j}\right)^{M_{j}-1} \Gamma\left(M_{j}\right)} \\
& =P\left(M_{j}-1, \frac{\gamma}{\bar{\gamma} \lambda_{j}}\right)
\end{aligned}
$$

The proof for $\gamma_{R j}$ is analogous and omitted here. 
Theorem 2. Given $\gamma_{t h}>0$ as the minimum SINR required at $S U_{j}$ to decode the received signal, the E2E OP $P_{j}^{\text {out }}\left(\gamma_{t h}\right)=$ $\operatorname{Pr}\left[S_{j} \leq \gamma_{t h}\right]$ at $S U_{j}(j=1,2)$ is given by $(8)$.

$$
\begin{aligned}
& P_{j}^{\text {out }}\left(\gamma_{t h}\right)=1-2 \sum_{i=2}^{M_{\bar{j}}} \sum_{k=0}^{M_{\bar{j}}-i} \sum_{l=0}^{k} \frac{e^{-\frac{\gamma_{t h}}{\bar{\gamma} \lambda_{\bar{j}}}\left(\frac{\gamma_{t h}}{\lambda_{\bar{j}}}\right)^{\frac{M_{1}+M_{2}-i+k-1}{2}}}}{\left(M_{j}-2\right) !\left(M_{\bar{j}}-i-k\right) !(k-l) !} \\
& \frac{\left(\frac{C}{\lambda_{j}}\right)^{\frac{M_{1}+M_{2}-i-k-1}{2}}\left(\gamma_{0} \lambda_{0}\right)^{l}}{\bar{\gamma}^{\left(M_{1}+M_{2}-i-l-2\right)}\left(\frac{\gamma_{t h} \gamma_{0} \lambda_{0}}{\lambda_{\bar{j}}}+\bar{\gamma}\right)^{l+1}} \mathcal{K}_{M_{j}-M_{\bar{j}}+i+k-1}\left(2 \sqrt{\frac{C \gamma_{t h}}{\bar{\gamma}^{2} \lambda_{1} \lambda_{2}}}\right)
\end{aligned}
$$

Proof. Since $f_{r} \sim \mathcal{C N}\left(0, \lambda_{0}\right), \gamma_{3}$ is $\operatorname{Exp}\left(\frac{1}{\gamma_{0} \lambda_{0}}\right)$-distributed.

Without loss of generality, due to the symmetric system setup, we derive the $\operatorname{CDF} F_{S_{1}}(\gamma)(\gamma>0)$ here. Since in Theorem 1, we have proved that $\gamma_{j R}$ and $\gamma_{R j}(j=1,2)$ are identically distributed, both are denoted as $\gamma_{j}$ here for simplicity. Then $F_{S_{1}}(\gamma)$ is derived as follows.

$$
F_{S_{1}}(\gamma)=\int_{0}^{\infty} \int_{0}^{\infty} F_{\gamma_{2}}\left(\left(\gamma_{3}+1+\frac{C}{\gamma_{1}}\right) \gamma\right) f_{\gamma_{3}}\left(\gamma_{3}\right) f_{\gamma_{1}}\left(\gamma_{1}\right) \mathrm{d} \gamma_{3} \mathrm{~d} \gamma_{1}
$$

By replacing $F_{\gamma_{2}}\left(\left(\gamma_{3}+1+\frac{C}{\gamma_{1}}\right) \gamma\right)$ in (9) with the expansion $P(s, x)=1-e^{-x} \sum_{t=0}^{s-1} \frac{x^{t}}{t !}$, given $s$ is a positive integer, we obtain (10), where the second equality follows the Binomial expansion. Then applying [6, Eq. (3.351.3)] and [6, Eq.(3.471.9)], the end-to-end OP (8) is derived.

Note that (8) includes only $\lambda_{i}(i=0,1,2,3)$. Therefore, only the location of the relay impacts the OP.

\section{B. Calculation of Fixed Relay Gain $G$}

Since (4) shows that the phase of $G$ does not matter, we assume $G$ is positive real valued. Then substituting $\boldsymbol{m}_{1}$ and $\boldsymbol{m}_{2}$ in Section II-B into (4), the relay gain is calculated as,

$$
G=\sqrt{\frac{I_{t h}}{\lambda_{3}\left[P_{s} \lambda_{1}\left(M_{1}-1\right)+P_{s} \lambda_{2}\left(M_{2}-1\right)+P_{p} \lambda_{0}+N_{0}\right]}},
$$

which results from the following two facts. First, because $\rho_{\boldsymbol{h}_{j}}(j=1,2)$ is Beta distributed with parameter $M_{j}-1$ and 1, and independent from both $\boldsymbol{g}_{j}$ and $\boldsymbol{h}_{j}$, it is true that $\mathbb{E}\left\{\left\|\boldsymbol{g}_{j}\right\|_{F}^{2} \rho_{\boldsymbol{h}_{j}}\right\}=\mathbb{E}\left\{\left\|\boldsymbol{g}_{j}\right\|_{F}^{2}\right\} \mathbb{E}\left\{\rho_{\boldsymbol{h}_{j}}\right\}$ and $\mathbb{E}\left\{\rho_{\boldsymbol{h}_{j}}\right\}=\frac{M_{j}-1}{M_{j}}$. Secondly, we have $\mathbb{E}\left\{\left|h_{r}\right|^{2}\right\}=\lambda_{3}, \mathbb{E}\left\{\left|f_{r}\right|^{2}\right\}=\lambda_{0}$ and $\mathbb{E}\left\{\left\|\boldsymbol{g}_{j}\right\|_{F}^{2}\right\}=M_{j} \lambda_{j}(j=1,2)$.

\section{E2E Outage Probability with $I_{t h} \rightarrow \infty$}

When $I_{t h} \rightarrow \infty$, the underlay network is equivalent to a conventional two-way relay network, where the secondary nodes can transmit freely and A is an interference source. Then, to derive the asymptotic E2E OP, we expand $\mathcal{K}_{\nu}(z)$ as,

$$
\begin{array}{r}
\mathcal{K}_{\nu}(z)=\frac{1}{2}\left(\frac{1}{2} z\right)^{-\nu} \sum_{p=0}^{\nu-1} \frac{(\nu-p-1) !}{p !}\left(-\frac{1}{4} z^{2}\right)^{p}+(-1)^{\nu+1} \\
\cdot\left(\frac{z}{2}\right)^{\nu} \sum_{p=0}^{\infty} \frac{\left(\frac{z^{2}}{4}\right)^{p}\left[\ln \left(\frac{z}{2}\right)-\frac{1}{2} \psi(p+1)-\frac{1}{2} \psi(\nu+p+1)\right]}{p !(\nu+k) !}
\end{array}
$$

where $\psi(x)$ is the diagamma function. Then, substitute $C=$ $\frac{\bar{\gamma}}{G^{2}}$ and (11) into (12) and define $\alpha_{1}=\frac{\lambda_{3}}{I_{t h}}\left[\lambda_{1}\left(M_{1}-1\right)+\right.$ $\left.\lambda_{2}\left(M_{2}-1\right)\right], \alpha_{2}=\frac{\lambda_{3}}{I_{t h}}\left(P_{p} \lambda_{0}+N_{0}\right)$, and $b=\frac{\gamma_{t h} N_{0}}{\lambda_{1} \lambda_{2}}\left(\alpha_{1}+\frac{\alpha_{2}}{P_{s}}\right)$. There are three cases: $(1)\left(M_{j}-1\right)-\left(M_{\bar{j}}-i-k\right)<0$,
(2) $\left(M_{j}-1\right)-\left(M_{\bar{j}}-i-k\right)=0$, and $(3)\left(M_{j}-1\right)-\left(M_{\bar{j}}-i-k\right)>$ 0 . Note that $\mathcal{K}_{\nu}(z)$ has the property that $\mathcal{K}_{\nu}(z)=\mathcal{K}_{-\nu}(z)$. And with $I_{t h} \rightarrow \infty, \mathcal{K}_{M_{j}-M_{\bar{j}}+i+k-1}\left(2 \sqrt{\frac{b}{I_{t h}}}\right)=0$ for case (1) and (2). For case (3), the second term in (12) converges to zero with $I_{t h} \rightarrow \infty$, and only the first term left.

Consequently, substituting $C=\frac{\bar{\gamma}}{G^{2}}$ and (11) into (8), it is found that the non-zero terms are only when $M_{\bar{j}}-i-k=0$ and $p=0$. Thus, the asymptotic E2E OP with $I_{t h} \rightarrow \infty$ is

$$
\begin{aligned}
& P_{j}^{\text {out }}\left(\gamma_{t h} ; I_{t h} \rightarrow \infty\right)=1 \\
& -\sum_{i=2}^{M_{\bar{j}}} \sum_{l=0}^{M_{\bar{j}}-i} \frac{e^{-\frac{\gamma_{t h} N_{0}}{P_{s} \lambda_{\bar{j}}}}\left(N_{0} \gamma_{t h}\right)^{M_{\bar{j}}-i}\left(\gamma_{0} \lambda_{0}\right)^{l}}{\lambda_{\bar{j}}^{M_{\bar{j}}-i} P_{s}^{M_{\bar{j}}-i}\left(\frac{P_{p} \lambda_{0} \gamma_{t h}}{P_{s} \lambda_{\bar{j}}}+1\right)^{(l+1)}\left(M_{\bar{j}}-i-l\right) !}
\end{aligned}
$$

As $I_{t h} \rightarrow \infty$, the E2E OP will converge to a constant (13) due to A's interference. Therefore, the diversity order is zero.

\section{E2E Outage Probability with $P_{s} \rightarrow \infty$}

In this section, we derive the asymptotic E2E OP in high transmit power region $\left(P_{s} \rightarrow \infty\right)$ for two cases: (1) when $I_{t h}$ is fixed and (2) $I_{t h}=a P_{s}, a>0$.

1) Fixed $I_{t h}$ : The asymptotic E2E OP $P_{j}^{\text {out }}\left(\gamma_{t h}\right)$ in this case is derived by substituting (11) into (8), and using the fact that with $P_{s} \rightarrow \infty, \alpha_{1}+\frac{\alpha_{2}}{P_{s}} \rightarrow \alpha_{1}, e^{-\frac{N_{0} \gamma_{t h}}{\lambda_{\bar{j}} P_{s}}} \rightarrow 1,\left(\frac{P_{p} \lambda_{0} \gamma_{t h}}{\lambda_{\bar{j}} P_{s}}+\right.$ 1) ${ }^{(l+1)} \rightarrow 1$, and the non-zero term in the sum is when $k=0$ and consequently $l=0$. Therefore, the E2E OP converges to

$$
\begin{aligned}
& P_{j}^{\text {out }}\left(\gamma_{t h} ; P_{s} \rightarrow \infty\right)=1-2 \sum_{i=2}^{M_{\bar{j}}} \frac{\left(\frac{\alpha_{1} \gamma_{t h} N_{0}}{I_{t h} \lambda_{1} \lambda_{2}}\right)^{\frac{M_{j}+M_{\bar{j}}-i-1}{2}}}{\left(M_{j}-2\right) !\left(M_{\bar{j}}-i\right) !} \\
& \cdot \mathcal{K}_{M_{j}-M_{\bar{j}}+i-1}\left(2 \sqrt{\frac{\alpha_{1} N_{0} \gamma_{t h}}{I_{t h} \lambda_{1} \lambda_{2}}}\right),
\end{aligned}
$$

which is a constant. Therefore, the diversity order is zero in this case.

2) $I_{t h}=a P_{s}$ : In this case, the underlay setup is equivalent to conventional two-way relay. We give the following theorem.

Theorem 3. If $\frac{I_{t h}}{P_{s}}=a,\left(\min \left\{M_{1}, M_{2}\right\}-1\right)$ diversity can be achieved in high transmit power region $\left(P_{s} \rightarrow \infty\right)$, and the corresponding asymptotic E2E OP is given by,

$$
P_{j}^{\text {out }}\left(\gamma_{t h} ; \frac{I_{\text {th }}}{P_{s}}=a, P_{s} \rightarrow \infty\right)=\phi\left(M_{1}, M_{2}\right)\left(\frac{b_{1}}{P_{s}}\right)^{\min \left(M_{1}, M_{2}\right)-1},
$$

where $b_{1}=\frac{N_{0} \gamma_{t h} \alpha_{1}}{a \lambda_{1} \lambda_{2}}$ and $\phi\left(M_{1}, M_{2}\right)$ is given in (16).

$$
\phi\left(M_{1}, M_{2}\right)= \begin{cases}-\sum_{i=1}^{M_{\bar{j}}} \frac{(-1)^{i-1}\left(M_{j}-M_{\bar{j}}-1\right) !}{\left(M_{\bar{j}}-i\right) !\left(M_{j}-2\right) !(i-1) !} & \text { if } M_{j}>M_{\bar{j}} \\ -\sum_{i=1}^{M_{\bar{j}}} \frac{(-1)^{i}\left[\ln \left(\frac{b_{1}}{P_{s}}\right)-\psi(1)-\psi(i)\right]}{\left(M_{\bar{j}}-i\right) !\left(M_{j}-2\right) !(i-1) !} & \text { if } M_{j}=M_{\bar{j}} \\ -\sum_{i=1}^{M_{\bar{j}}} \varphi(i) & \text { if } M_{j}<M_{\bar{j}}\end{cases}
$$

$\varphi(i)= \begin{cases}\frac{(-1)^{M_{j}-M_{\bar{j}}+i}\left[\ln \left(\frac{b_{1}}{P_{s}}\right)-\psi(1)-\psi\left(M_{j}-M_{\bar{j}}+i\right)\right]}{\left(M_{j}-1\right) !\left(M_{\bar{j}}-i\right) !\left(M_{j}-M_{\bar{j}}+i-1\right) !} & \text { if } M_{j}-1>M_{\bar{j}}-i \\ \frac{2 \psi(1)-\ln \left(\frac{b_{1}}{P_{s}}\right)}{\left(M_{j}-1\right) ! !\left(M_{\bar{j}}-i\right) !} & \text { if } M_{j}-1=M_{\bar{j}}-i \\ \frac{\left(M_{\bar{j}}-M_{j}-i\right) !}{\left(M_{j}-2\right) !\left(M_{\bar{j}}-i\right) !} & \text { if } M_{j}-1<M_{\bar{j}}-i\end{cases}$

Proof. Without loss of generality, the $\mathrm{OP}$ at $\mathrm{SU}_{1}$ is used here.

Substituting $I_{t h}=a P_{s}$ into (14) results in (17).

If $M_{1} \geq M_{2}, M_{1}-1-M_{2}+i>0$ always holds. Then after applying the expansion (12) of $\mathcal{K}_{v}(\cdot)$, the lowest order of 


$$
\begin{aligned}
& F_{S_{1}}(\gamma)=1-\sum_{i=2}^{M_{2}} \int_{0}^{\infty} \int_{0}^{\infty} \frac{e^{-\left(\gamma_{3}+1+\frac{C}{\gamma_{1}}\right) \frac{\gamma}{\lambda_{2} \bar{\gamma}}\left(\frac{\gamma}{\bar{\gamma} \lambda_{2}}\right)^{M_{2}-i} \gamma_{1}^{M_{1}-2}}}{\left(M_{1}-2\right) !\left(M_{2}-i\right) !\left(\bar{\gamma} \lambda_{1}\right)^{M_{1}-1} \gamma_{0} \lambda_{0}}\left(\gamma_{3}+1+\frac{C}{\gamma_{1}}\right)^{M_{2}-i} e^{-\frac{\gamma_{3}}{\gamma_{0} \lambda_{0}}} e^{-\frac{\gamma_{1}}{\gamma \lambda_{1}}} \mathrm{~d} \gamma_{3} \mathrm{~d} \gamma_{1}
\end{aligned}
$$

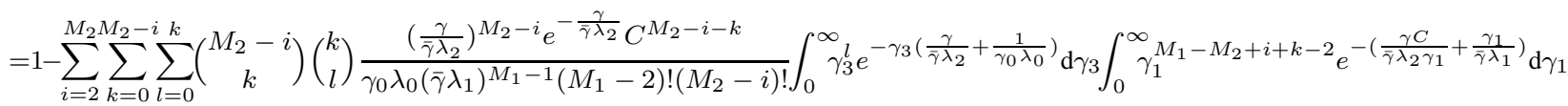

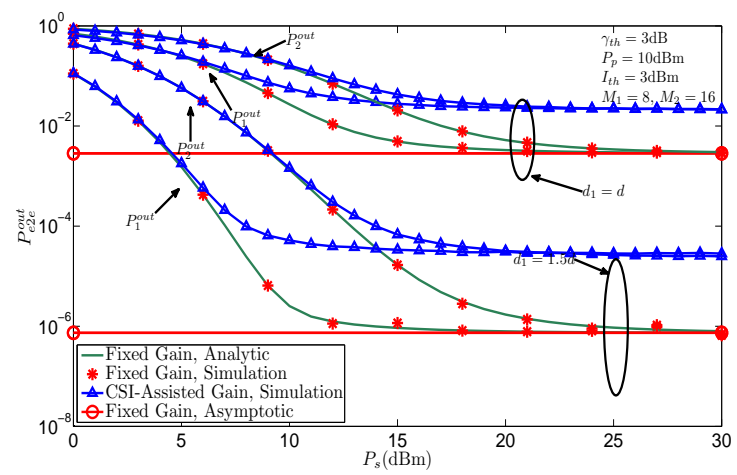

Fig. 2. $P_{e 2 e}^{\text {out }}$ v.s. $P_{s}$ with $I_{t h}=3 \mathrm{dBm}$ and $M_{1}=8, M_{2}=16$

$\frac{1}{P_{s}}$ is given by $\left(M_{2}-1\right)$ when $M_{1}=M_{2}$ and $p$ in the second term of (12) is zero. If $M_{1}>M_{2}$, the lowest order of $\frac{1}{P_{s}}$ is given by $\left(M_{2}-1\right)$ when $p$ in the first term of (12) takes value of $i-1$. If $M_{1}<M_{2}$, when $M_{1}-1-M_{2}+i \geq 0$, the lowest order of $\frac{1}{P_{s}}$ is given by $\left(M_{1}-1\right)$ with $p$ in the second term of (12) being zero. When $M_{1}-1-M_{2}+i<0$, using the $\mathcal{K}_{v}(\cdot)=\mathcal{K}_{-v}(\cdot)$ property, the lowest order of $\frac{1}{P_{s}}$ is given by $\left(M_{2}-1\right)$ with $p$ in the first term of (12) being $i-1$.

$$
P_{1}^{\text {out }}\left(\gamma_{t h}\right)=1-2 \sum_{i=2}^{M_{2}} \frac{\left(\frac{b_{1}}{P_{s}}\right)^{\frac{M_{1}+M_{2}-i-1}{2}}}{\left(M_{1}-2\right) !\left(M_{2}-i\right) !} \mathcal{K}_{M_{1}-M_{2}+i-1}\left(2 \sqrt{\frac{b_{1}}{P_{s}}}\right) \text {. }
$$

Comparatively, the underlay setup is equivalent to the conventional two-way relay networks consisting of two multiantenna terminals and one single-antenna fixed gain AF relay [9]. In [9], the two terminals use MRT/MRC and a diversity of order $\min \left(M_{1}, M_{2}\right)$ is achieved in high transmit power region. But in our study, ZFB-MRT/MRC applied at the two terminals achieves reduced diversity order (Theorem 3), which is due to orthogonality requirement to enforce ZFB resulting in a small loss of degrees of freedom.

\section{NumERICAL RESUlTS}

This section provides numerical results to validate the preceding analysis. The parameters $N_{0}, P_{p}$ and $\gamma_{t h}, \omega_{\mathrm{x}, \mathrm{y}}$ are set to $0 \mathrm{~dB}, 10 \mathrm{dBm}$ and $3 \mathrm{~dB}, 3.5$ respectively. We also assume that $d_{\mathrm{SU}_{j}, \mathrm{R}}=d(j=1,2)$ and $d_{\mathrm{A}, \mathrm{R}}=d_{\mathrm{R}, \mathrm{B}}=d_{1}$.

Fig. 2 shows the E2E OPs as a function of $P_{s}$ with $d_{1}=d$ and $d_{1}=1.5 d$. For comparisons, simulation results of CSIAssisted gain are provided as well, where $\mathrm{R}$ adapts gain as $G=\sqrt{\frac{I_{t h}}{\left|h_{r}\right|^{2}\left[P_{s}\left|\boldsymbol{g}_{1}^{T} \boldsymbol{m}_{1}\right|^{2}+P_{s}\left|\boldsymbol{g}_{2}^{T} \boldsymbol{m}_{2}\right|^{2}+P_{p}\left|f_{r}\right|^{2}+N_{0}\right]}}$. It is shown that the E2E OPs of CSI-Assisted gain overlap those of fixed gain in lower $P_{s}$ region, e.g. $P_{s} \leq 5 \mathrm{dBm}$. With increasing $P_{s}$, fixed gain outperforms CSI-Assisted gain. This is because with CSI-Assisted gain, $G$ is chosen such that the interference power at B below $I_{t h}$ in each transmission, while fixed gain considers the average interference power. It is also shown that when R-A/B distance is larger (1.5d), lower OP is achieved.

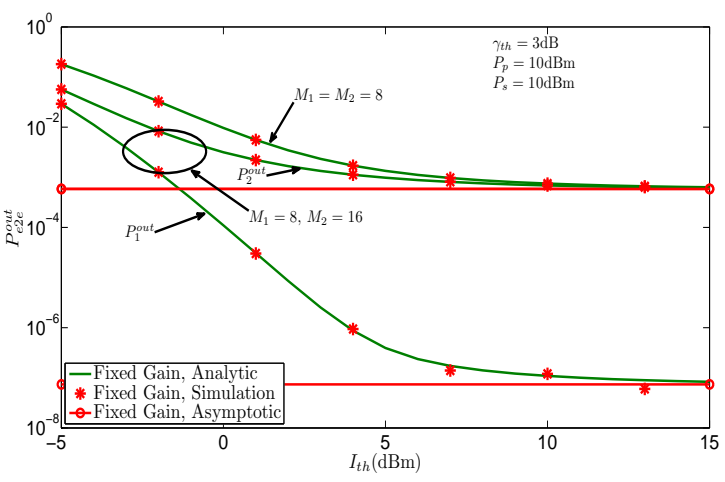

Fig. 3. $P_{e 2 e}^{o u t}$ v.s. $I_{t h}$ with $d_{1}=1.5 d$ and $P_{s}=10 \mathrm{dBm}$

Fig. 3 plots a E2E OPs as the function of $I_{t h}$ for $M_{1}=$ $M_{2}=8$ and $M_{1}=8, M_{2}=16$, respectively. With increasing $I_{t h}$, the OPs at $\mathrm{SU}_{2} \mathrm{~S}$ in both setups converge to the same OP floor since both $\mathrm{SU}_{1} \mathrm{~s}$ are equipped with 8 antennas.

\section{CONCLUSION}

The exact and asymptotic OPs of an underlay multi-antenna network with a two-way relay has been analysed. Both path loss effect and Rayleigh fading were considered, and ZFBMRT/MRC were used to mitigate interference effects. The location of the relay is shown to significantly impact OP. As $P_{s} \rightarrow \infty$, the diversity order is zero if $I_{t h}$ is finite, but if $\frac{I_{t h}}{P_{s}}$ is constant, $\left(\min \left(M_{1}, M_{2}\right)-1\right)$ diversity is achieved.

\section{REFERENCES}

[1] Z. Dai, J. Liu, C. Wang, and K. Long, "An adaptive cooperation communication strategy for enhanced opportunistic spectrum access in cognitive radios," IEEE Commun. Lett., vol. 16, no. 1, pp. 40-43, Jan. 2012.

[2] A. Afana, V. Asghari, A. Ghrayeb, and S. Affes, "On the performance of cooperative relaying spectrum-sharing systems with collaborative distributed beamforming," IEEE Trans. Commun., vol. 62, no. 3, pp. 857871, Mar. 2014.

[3] T. M. Chinh Chu, H. Phan, T. Q. Duong, M. Elkashlan, and H.-J. Zepernick, "Beamforming transmission in cognitive AF relay networks with feedback delay," in Proc. Int. Conf. Comput., Manage. Telecommun. (ComManTel), Jan. 2013, pp. 117-122.

[4] N. Yang, P. Yeoh, M. Elkashlan, I. Collings, and Z. Chen, "Twoway relaying with multi-antenna sources: Beamforming and antenna selection," IEEE Trans. Veh. Technol., vol. 61, no. 9, pp. 3996-4008, Nov. 2012.

[5] H. Katiyar and R. Bhattacharjee, "Performance of regenerative relay network operating in uplink of multi-antenna base station under Rayleigh fading channel," in Proc. IEEE TENCON 2009, Jan. 2009, pp. 1-5.

[6] I. Gradshteyn and I. Ryzhik, Table of Integrals, Series, and Products. Academic Press, 2007.

[7] T. Cui, C. Tellambura, and Y. Wu, "Low-complexity pilot-aided channel estimation for OFDM systems over doubly-selective channels," in 2005 IEEE Int. Conf. Commun. (ICC), vol. 3, 2005, pp. 1980-1984.

[8] J. Yang, S. Jang, and D. K. Kim, "Sum rate approximation of zeroforcing beamforming with semi-orthogonal user selection," J. Commun. and Networks, vol. 12, no. 3, pp. 222-230, Jun. 2010.

[9] T. Duong, H. Suraweera, H. Zepernick, and C. Yuen, "Beamforming in two-way fixed gain amplify-and-forward relay systems with CCI," in 2012 IEEE Int. Conf. Commun. (ICC), Jun. 2012, pp. 3621-3626. 